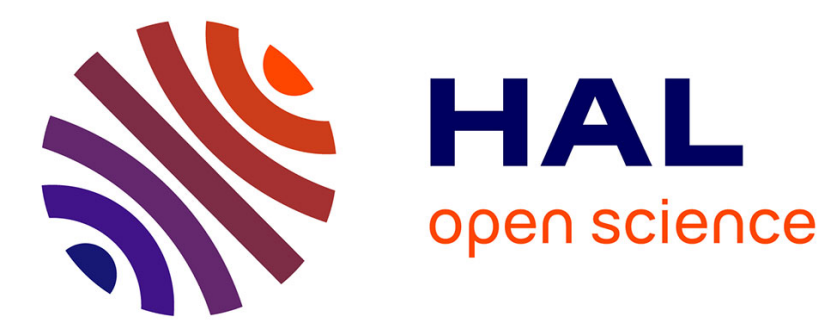

\title{
Stability analysis of a wastewater treatment plant with saturated control
}

Frédéric Grognard, Olivier Bernard

\section{To cite this version:}

Frédéric Grognard, Olivier Bernard. Stability analysis of a wastewater treatment plant with saturated control. Water Science and Technology, 2006, 53 (1), pp.149-157. 10.2166/wst.2006.017 . hal01090322

\section{HAL Id: hal-01090322 \\ https://hal.inria.fr/hal-01090322}

Submitted on 3 Dec 2014

HAL is a multi-disciplinary open access archive for the deposit and dissemination of scientific research documents, whether they are published or not. The documents may come from teaching and research institutions in France or abroad, or from public or private research centers.
L'archive ouverte pluridisciplinaire HAL, est destinée au dépôt et à la diffusion de documents scientifiques de niveau recherche, publiés ou non, émanant des établissements d'enseignement et de recherche français ou étrangers, des laboratoires publics ou privés. 


\title{
Stability analysis of a wastewater treatment plant with saturated control
}

\author{
F. Grognard and O. Bernard \\ INRIA Sophia-Antipolis, Projet COMORE, 2004 route des Lucioles, BP 93, 06902 Sophia-Antipolis \\ Cedex, FRANCE. (E-mail: frederic.grognard@inria.fr, olivier.bernard@inria.fr)
}

\begin{abstract}
This paper presents a saturated proportional controller that achieves depollution of wastewater in a continuous anaerobic digester. This goal is reached by defining a region of the state-space where the depollution is achieved and forcing attractivity and invariance of this region. The control variable is the dilution rate and the controlled variable is a linear combination $\left(S_{\lambda}\right)$ of the substrates concentrations, that could be the Chemical Oxygen Demand (COD) or the Biological Oxygen Demand (BOD), depending on the value of $\lambda$. No measurement of the substrates concentrations in the input flow is required; the only necessary measurement is $S_{\lambda}$.
\end{abstract}

Keywords

Anaerobic digestion, bioreactor, COD, control, saturation.

\section{INTRODUCTION}

The control of bioreactors is a delicate problem since most of the time the available biological models are only rough approximations, the biological systems being known to be highly variable and difficult to measure. To circumvent this difficulty, Bastin and Dochain (1990) have introduced the mass balance based modelling. The main idea of this approach is to design estimators and controllers independently of any modelling of the biological kinetics.

Among the bioreactors, those dedicated to wastewater treatment especially suffer from the modelling uncertainties. A complex ecosystem composed by many different bacterial populations takes place in these processes, and the composition and concentration of the pollutant to degrade is not well known and evolves with respect to time. Moreover, most of the time no measurement of the involved chemical or biological species is available; this can be critical when the bioreactor is unstable as is the case for the anaerobic digestor. In these conditions, a control procedure that would guarantee the process stability should be as insensitive as possible to all these parameters.

In this paper, we will consider an anaerobic wastewater treatment process, that is a biological process in which biodegradable organic materials are decomposed in the absence of oxygen to produce methane. The underlying model assumes that two main bacterial populations are present (Bernard et al. 2001). The first one, the acidogenic bacteria $X_{1}$, consumes the organic substrate $S_{1}$ and produces through an acidogenesis step volatile fatty acids (VFA) $S_{2}$. The second population (methanogenic bacteria) $X_{2}$, uses the VFA in a methanogenesis step as substrate for growth and produces methane.

Despite its capacity to degrade difficult substrates, this process is known to become unstable under certain circumstances, like variations of the process operating conditions, and requires therefore a monitoring procedure to detect a destabilization. This must also be associated to a control action that can avoid the risk of acidification of the fermenter. Therefore, some control laws have recently been introduced for this process like the adaptive feedback of the gaseous flow-rate measurement (Perrier and Dochain 1993, Mailleret et al. 2003, Mailleret et al. 2004) or fuzzy control of the VFA concentration (Genovesi et al. 1999, Punal et al. 2000) to avoid acidification of the reactor. The controller that we have designed regulates a linear combination of the substrates concentrations, that we will denote 
$S_{\lambda}$; depending on the value of the parameter $\lambda, S_{\lambda}$ can represent the Biological Oxygen Demand (BOD) or the Chemical Oxygen Demand (COD), that is the standard measurement of the pollution level. Our controller requires the measurement, or the observation through software sensors (through the application of techniques similar to (Alcaraz-Gonzalez et al. 2002)), of $S_{\lambda}$ and has a very simple structure that takes actuator limitations into account (as is also done in Antonelli et al. (2003)); it has the advantage of not requiring any measurement of the substrates concentrations in the input flow. The variable that is used for control is the dilution rate $(D)$.

\section{MODEL OF ANAEROBIC DIGESTION}

In this paper, we will use the model AM1 of anaerobic digestion that was presented in (Bernard et al. 2001):

$$
\left\{\begin{array}{l}
\dot{X}_{1}=\left(\mu_{1}\left(S_{1}\right)-\alpha D\right) X_{1} \\
\dot{X}_{2}=\left(\mu_{2}\left(S_{2}\right)-\alpha D\right) X_{2} \\
\dot{S}_{1}=D\left(S_{1 i n}-S_{1}\right)-k_{1} \mu_{1}\left(S_{1}\right) X_{1} \\
\dot{S}_{2}=D\left(S_{2 i n}-S_{2}\right)+k_{2} \mu_{1}\left(S_{1}\right) X_{1}-k_{3} \mu_{2}\left(S_{2}\right) X_{2}
\end{array}\right.
$$

with $X_{1}, X_{2}, S_{1}, S_{2}, D \in \mathbb{R}^{+}, \mu_{1}\left(S_{1}\right)$ a non-decreasing and bounded function such that

$$
\mu_{1}(0)=0 \text { and } \mu_{1}\left(S_{1}\right)<\mu_{1 \max } \forall S_{1} \geq 0
$$

and $\mu_{2}\left(S_{2}\right)$ a function such that

$$
\mu_{2}(0)=0 \text { and } \mu_{2}\left(S_{2}\right) \leq \mu_{2 \max }=\mu_{2}\left(S_{2}^{*}\right) \forall S_{2} \geq 0
$$

and $\mu_{2}\left(S_{2}\right)$ is non-decreasing from $S_{2}=0$ to $S_{2}=S_{2}^{*}$ and non-increasing afterwards with

$$
\lim _{S_{2} \rightarrow+\infty} \mu_{2}\left(S_{2}\right)=0
$$

Classically, $\mu_{1}$ is of the Monod type and $\mu_{2}$ of the Haldane type. The terms $S_{1 i n}$ and $S_{2 i n}$ are the influent concentrations of $S_{1}$ and $S_{2}$ respectively. The $k_{i}$ represent the yield coefficients associated with bacterial growth. The parameter $\alpha \in[0,1]$ represents the proportion of bacteria that are not fixed on the bed, and therefore that are affected by the dilution effect: $\alpha=0$ would correspond to an ideal fixed bed reactor, $\alpha=1$ to an ideal continuous stirred tank reactor. This model has been built and validated with the spirit of finding a trade-off between model complexity and mathematical handling of the model for control purpose. It is not intended at giving an accurate view of all the phenomena that take place in the reactor as higher-dimensional models do (e.g.the IWA Anaerobic Digestion Model No.1 (Batstone et al. 2002)).

\section{OBJECTIVE AND CONSTRAINTS}

The original control objective for depollution is to regulate the output $S_{\lambda}=S_{1}+\lambda S_{2}$ (with $\lambda \geq 0$ not always equal to 1 because $S_{1}$ and $S_{2}$ do not need to be expressed in the same units), which, depending on the chosen value for $\lambda$, can be the COD or BOD. The target value for $S_{\lambda}$ is some $\bar{S}_{\lambda} \leq S_{\lambda \max } \leq S_{\lambda i n}=S_{1 i n}+\lambda S_{2 i n}$. In this paper, the objective is modified as follows

Objective 1 Given $S_{\lambda \min } \leq \bar{S}_{\lambda} \leq S_{\lambda \max }$, steer all the solutions of the controlled system to a region where $S_{\lambda \min } \leq S_{\lambda} \leq S_{\lambda \max }$ is satisfied and stays valid for all future times

Instead of achieving regulation, we will achieve attractivity and invariance of a security zone. In this formulation, $S_{\lambda \max }$ is an unalterable data of the problem (fixed by depollution norms); on the other hand, $S_{\lambda \min }$ can be chosen more freely: if it is taken close to $S_{\lambda \max }$, the achievement of Objective 1 is almost equivalent to the regulation of the output $S_{\lambda}$; if $S_{\lambda \min }$ is taken close to zero, there is a risk 
that the system settles at a small value of $S_{\lambda}$ with a small value of the dilution rate. The pollutant concentrations in the input, $S_{1 i n}$ and $S_{2 i n}$, are supposed to be constant. They do not need to be known for the application of the controller. However, in order to show stability of the controller, those values need to be known.

In order to design a controller, we first analyze the different parameters associated to the control objective. In the sequel, we will show that the following assumption needs to be imposed.

Assumption 1 The parameters satisfy the following three inequalities

$$
\begin{gathered}
\lambda<\frac{k_{1}}{k_{2}} \\
S_{\lambda \max }<\min \left(S_{1 i n}, \lambda T_{2 i n}\right)=\min \left(S_{1 i n}, \frac{\lambda k_{2}}{k_{1}} S_{1 i n}+\lambda S_{2 i n}\right) \\
D_{\max }<\frac{\min \left(\mu_{1}\left(S_{1 i n}\right), \mu_{2}\left(S_{2 i n}\right), \mu_{2}\left(T_{2 i n}\right)\right)}{\alpha}
\end{gathered}
$$

\section{The parameter $\lambda$}

The evolution of the pollution level follows the following equation:

$$
\dot{S}_{\lambda}=D\left(S_{\lambda i n}-S_{\lambda}\right)-\left(k_{1}-\lambda k_{2}\right) \mu_{1}\left(S_{1}\right) X_{1}-\lambda k_{3} \mu_{2}\left(S_{2}\right) X_{2}
$$

Condition (2) imposes that the pollution level decreases when the flow rate is stopped (which is the intuitive behavior of a digester). This condition is met by the identified parameters of the experimental process (Bernard et al. 2001) when $S_{\lambda}$ is the COD $\left(\lambda=0.064 \mathrm{~g} / \mathrm{mmol}\right.$ and $\left.\frac{k_{1}}{k_{2}}=0.368 \mathrm{~g} / \mathrm{mmol}\right)$.

The bound $S_{\lambda \max }$

In the rest of this section, we will replace $S_{2}$ with the coordinate $T_{2}=S_{2}+\frac{k_{2}}{k_{1}} S_{1}$. This results in the following system:

$$
\left\{\begin{array}{l}
\dot{X}_{1}=\left(\mu_{1}\left(S_{1}\right)-\alpha D\right) X_{1} \\
\dot{X}_{2}=\left(\mu_{2}\left(T_{2}-\frac{k_{2}}{k_{1}} S_{1}\right)-\alpha D\right) X_{2} \\
\dot{S}_{1}=D\left(S_{1 i n}-S_{1}\right)-k_{1} \mu_{1}\left(S_{1}\right) X_{1} \\
\dot{T}_{2}=D\left(T_{2 i n}-T_{2}\right)-k_{3} \mu_{2}\left(T_{2}-\frac{k_{2}}{k_{1}} S_{1}\right) X_{2}
\end{array}\right.
$$

considered in the positively invariant set $\left\{\left(X_{1}, X_{2}, S_{1}, T_{2}\right) \in \mathbb{R}_{+}^{4} \mid T_{2} \geq \frac{k_{2}}{k_{1}} S_{1}\right\}$.

In these new variables, the measure $S_{\lambda}$ is rewritten as $S_{\lambda}=S_{1}+\lambda S_{2}=\left(1-\lambda \frac{k_{2}}{k_{1}}\right) S_{1}+\lambda T_{2}$. We will now impose a condition that we will call "regulability": this condition makes sure that, whatever the level $\bar{S}_{\lambda} \leq S_{\lambda \max }$ that is regulated, there corresponds a non trivial equilibrium for system (6). If $S_{\lambda}$ is set at some prespecified value $\bar{S}_{\lambda}$, there should exist a constant dilution $\bar{D}>0$ corresponding to an equilibrium. From the $\dot{X}_{i}=0$ equations, we see that such an equilibrium should satisfy:

$$
\mu_{1}\left(\bar{S}_{1}\right)=\mu_{2}\left(\frac{\bar{S}_{\lambda}-\bar{S}_{1}}{\lambda}\right)>0
$$

This potentially results in several values of $\bar{S}_{1}>0$ for our equilibrium, and corresponding values of $\bar{D}$. Introducing this into the $\dot{S}_{1}=\dot{T}_{2}=0$ equations, we obtain

$$
\begin{aligned}
& 0=\left(S_{1 i n}-\bar{S}_{1}\right)-k_{1} \alpha \bar{X}_{1} \\
& 0=\left(T_{2 i n}-\bar{T}_{2}\right)-k_{3} \alpha \bar{X}_{2}
\end{aligned}
$$


Isolating $\bar{X}_{1}$ and $\bar{X}_{2}$, we get $\bar{X}_{1}=\frac{S_{1 i n}-\bar{S}_{1}}{k_{1} \alpha}$ and $\bar{X}_{2}=\frac{T_{2 i n}-\bar{T}_{2}}{k_{3} \alpha}$. At the equilibrium, $\bar{X}_{1}$ and $\bar{X}_{2}$ should be positive. Noticing that $\bar{S}_{1} \leq \bar{S}_{\lambda}<S_{\lambda \max }$ and $\lambda \bar{T}_{2} \leq \bar{S}_{\lambda}<S_{\lambda \max }$, it suffices to impose (3) to have $\bar{X}_{1}$ and $\bar{X}_{2}$ positive at any equilibrium having $\bar{S}_{\lambda}<S_{\lambda \max }$. This assumption also forces $S_{\lambda \max }<S_{\lambda i n}$; it is reasonable, as we want to bring pollution to a lower level than its influent value.

\section{Bounded control}

The control variable is the dilution rate, so that it must be non-negative, and it cannot be arbitrarily high. There is an a priori upper-bound on the maximal flow-rate $D_{\max }$ due to the physical constraint associated to the pumping mechanism. This bound can be seen as a given data, but it can also be seen as a design parameter (a different choice of input valve can give a different value of upper-bound for $D_{\max }$ ). On the other hand, the minimal value of the flow-rate is, theoretically, zero; however, in the industrial environment, the output of the industrial plant that produces the waste cannot be totally stopped, it is lower-bounded by some $D_{\min }>0$. We will design a controller that satisfies both these bounds. Moreover, equation (4) is imposed to avoid a wash-out of the bacteria of the reactor (we do not prove this property due to space limitation).

\section{Bounded state}

Based on that assumption and for $D_{\min }$ and $D_{\max }$ fixed, it can be shown that the solutions are bounded: there exist $S_{1 \min }, T_{2 \min }>0$ such that, for any controller $D_{\min } \leq D\left(X_{1}, S_{1}, X_{2}, S_{2}\right) \leq$ $D_{\max }$ and for any initial condition in the positive orthant $\left(X_{1}(0), S_{1}(0), X_{2}(0), S_{2}(0)\right) \in \mathbb{R}_{+}^{4}$, there exists a finite time $T>0$ after which the following four inequalities are valid for all $t \geq T$ :

$$
\begin{gathered}
S_{1 \text { in }}<k_{1} X_{1}(t)+S_{1}(t)<\frac{S_{1 i n}}{\alpha}, T_{2 i n}<k_{3} X_{2}(t)+T_{2}(t)<\frac{T_{2 i n}}{\alpha} \\
S_{1 \text { min }}<S_{1}(t)<S_{1 \text { in }}, T_{2 \text { min }}<T_{2}(t)<T_{2 \text { in }}
\end{gathered}
$$

These inequalities are not proven here due to space limitation: they are a consequence of the differentiation of the quantities $k_{1} X_{1}+S_{1}, k_{3} X_{2}+T_{2}, S_{1}$ and $T_{2}$ and result in the following lemma

Lemma 1 Let $0<D_{\min }<D_{\max }$ be fixed. Then, for any initial condition $\left(X_{1}(0), X_{2}(0), S_{1}(0), S_{2}(0)\right)$ belonging to $\mathbb{R}_{+}^{4}$, and for given constants $S_{1 i n}, S_{2 i n}$ such that Assumption 1 is satisfied, there exists a time $T>0$ such that, for all $t \geq T$, we have

$$
\begin{aligned}
& X_{1 m i n}<X_{1}(t)<\frac{S_{1 i n}}{k_{1} \alpha} \\
& 0<X_{2}(t)<\frac{T_{2 i n}}{k_{3} \alpha} \\
& S_{1 \text { min }}<S_{1}(t)<S_{1 \text { in }} \\
& T_{2 \min }<T_{2}(t)<T_{2 i n}
\end{aligned}
$$

along the solution of (1) for any choice of $D(t) \in\left[D_{\min }, D_{\max }\right]$.

\section{CONTROL DESIGN}

We choose a simple proportional controller in the form

$$
D=\frac{D_{\max }-D_{\min }}{2}\left(1+\operatorname{sat}\left(\frac{S_{\lambda \max }+S_{\lambda \min }-2 S_{\lambda}}{S_{\lambda \max }-S_{\lambda \min }}\right)\right)+D_{\min }
$$

where $\operatorname{sat}(s)=\frac{s}{\max (|s|, 1)}$ (the controller is illustrated on Figure 1). As stated in Objective 1, this controller is not designed to regulate $S_{\lambda}$ at a prespecified value $\bar{S}_{\lambda}$, but rather to ensure attractivity and invariance of the region of the state space where $S_{\lambda}$ belongs to an interval $\left[S_{\lambda \min }, S_{\lambda \max }\right]$. Such a controller should be more robust than a controller aimed at exactly regulating the output. The main 


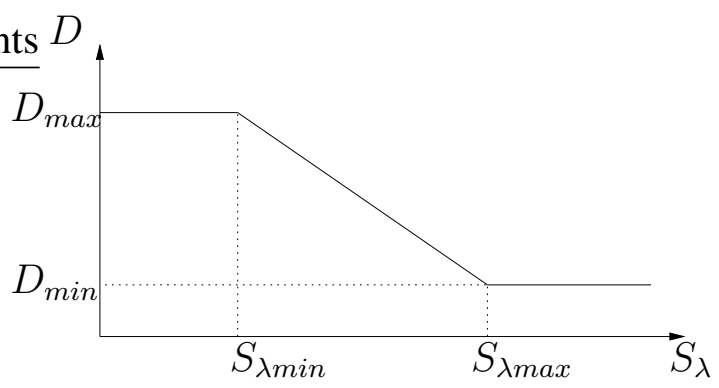

Figure 1: Form of the controller (9)

tuning parameters of this controller are the constants $D_{\max }$ and $S_{\lambda \min }$ (though $D_{\max }$ might not be picked arbitrarily large in the actual plant due to physical constraints).

This controller is based on the following philosophy:

(i) if $S_{\lambda} \geq S_{\lambda \max }$ then the flow is minimal: it prevents the pollution from leaving the plant in too large an amount; the pollution is lowered inside the plant and the bacteria grow in order to face the higher depollution requirement;

(ii) if $S_{\lambda} \leq S_{\lambda \min }$ then the flow is allowed to be maximal because the pollution level is low enough to be certain that this maximal flow will not drive the system into the region where the pollution is too high;

(iii) if $S_{\lambda \min }<S_{\lambda}<S_{\lambda \max }$ then the controller is linear and built such that it is continuous at the boundaries of this region.

The description of the controller as (i)-(ii)-(iii) allows for the separate description of the controlled system (1)-(9) in the three corresponding regions, that we will name $\Omega_{1}, \Omega_{2}$, and $\Omega_{3}$, respectively:

$\underline{\text { Region } \Omega_{1}: D=D_{\min }}$ The region $\Omega_{1}$ is defined as

$$
\Omega_{1}=\left\{\left(X_{1}, X_{2}, S_{1}, S_{2}\right) \in\left(\mathbb{R}^{+}\right)^{4} \mid S_{1}+\lambda S_{2} \geq S_{\lambda \max }\right\}
$$

In this region, where $S_{\lambda} \geq S_{\lambda \max }$, the flow rate is rendered minimal to limit the outflow of pollutants. System (1) can be rewritten as

$$
\left\{\begin{array}{l}
\dot{X}_{1}=\left(\mu_{1}\left(S_{1}\right)-\alpha D_{\text {min }}\right) X_{1} \\
\dot{X}_{2}=\left(\mu_{2}\left(S_{2}\right)-\alpha D_{\text {min }}\right) X_{2} \\
\dot{S}_{1}=D_{\text {min }}\left(S_{1 i n}-S_{1}\right)-k_{1} \mu_{1}\left(S_{1}\right) X_{1} \\
\dot{S}_{2}=D_{\text {min }}\left(S_{2 i n}-S_{2}\right)+k_{2} \mu_{1}\left(S_{1}\right) X_{1}-k_{3} \mu_{2}\left(S_{2}\right) X_{2}
\end{array}\right.
$$

This system can be analyzed as a cascade system between the $\left(X_{1}, S_{1}\right)$ subsystem and the $\left(X_{2}, S_{2}\right)$ subsystem. For any constant $D_{\min }<\frac{\mu_{1 \max }}{\alpha}$, the state of the $\left(X_{1}, S_{1}\right)$ subsystem globally converges to the non-trivial equilibrium $\left(\bar{X}_{1}, \bar{S}_{1}\right)=\left(\frac{S_{1 i n}-\mu_{1}^{-1}\left(\alpha D_{\min }\right)}{k_{1} \alpha}, \mu_{1}^{-1}\left(\alpha D_{\min }\right)\right)$. Also, the smaller $D_{\min }$ is, the smaller $\bar{S}_{1}$ is. Because the solutions of the whole system are bounded, we know that the behavior of the whole system (10) can be deduced from the behavior of the $\left(X_{2}, S_{2}\right)$ subsystem on the manifold $\left(X_{1}, S_{1}\right)=\left(\bar{X}_{1}, \bar{S}_{1}\right)$. This system is

$$
\left\{\begin{array}{l}
\dot{X}_{2}=\left(\mu_{2}\left(S_{2}\right)-\alpha D_{m i n}\right) X_{2} \\
\dot{S}_{2}=D_{\min }\left(\tilde{S}_{2 i n}-S_{2}\right)-k_{3} \mu_{2}\left(S_{2}\right) X_{2}
\end{array}\right.
$$


Generically, this system has two non-trivial equilibria because $\mu_{2}$ is similar to an Haldane function; the equilibria are characterized by the two values of $S_{2}$ that are such that $\mu_{2}\left(S_{2}\right)=\alpha D_{\min }\left(S_{2}^{m}<S_{2}^{*}<\right.$ $\left.S_{2}^{M}\right)$. It is straightforward to show that $S_{2}^{M}$ is an unbounded increasing function $D_{\text {min }}$. Independently of the choice of $D_{\text {min }}$, Lemma 1 shows that $S_{2} \leq T_{2} \leq T_{2 i n}$ after a finite time. Also, if we take $D_{\min }$ small enough, we can have $S_{2}^{M}>T_{2 i n}$, so that no convergence to the equilibrium corresponding to $S_{2}=S_{2}^{M}$ can take place and all solutions converge towards the equilibrium corresponding to $S_{2}=S_{2}^{m}$. This equilibrium is characterized by $\bar{S}_{\lambda}=\bar{S}_{1}+\lambda S_{2}^{m}=\mu_{1}^{-1}\left(\alpha D_{\text {min }}\right)+\lambda S_{2}^{m}$, which can be made as small as we want by reducing $D_{\text {min }}$. This ensures that system (10) has a single equilibrium, and that this equilibrium lies in the region where $S_{\lambda}<S_{\lambda \max }$. We can show that this equilibrium is attractive for all initial conditions for system (10), so that we know that $S_{\lambda}=S_{\lambda \max }$ is reached in finite time. We have then shown attractivity of $\Omega_{2} \cup \Omega_{3}$ for $D_{\min }$ small enough. We now have to show invariance of this set. On its border, (5) becomes:

$$
\dot{S}_{\lambda}=D_{\min }\left(S_{\lambda i n}-S_{\lambda \max }\right)-\left(k_{1}-\lambda k_{2}\right) \mu_{1}\left(S_{1}\right) X_{1}-\lambda k_{3} \mu_{2}\left(S_{2}\right) X_{2}
$$

We can show that, in the region defined by the constraints (7)-(8), we have $\left(k_{1}-\lambda k_{2}\right) \mu_{1}\left(S_{1}\right) X_{1}+$ $\lambda k_{3} \mu_{2}\left(S_{2}\right) X_{2} \geq M$ when $S_{\lambda}=S_{\lambda \max }$ for some $M>0$. This shows that, for $D_{\min }>0$ small enough $\dot{S}_{\lambda}<0$ when $S_{\lambda}=S_{\lambda \max }$. We then see that, as long as $D_{\min }$ is small enough, the region $\Omega_{2} \cup \Omega_{3}$ is attractive and invariant. We then state the following assumption to deduce Lemma 2 :

Assumption 2 The minimal dilution rate $D_{\text {min }}>0$ is taken small enough.

where the exact extent of the "small enough" term is defined in the attractivity and invariance conditions stated before this assumption.

Lemma 2 Under Assumptions 1 and 2, there exists a finite time $T$ after which the region $\Omega_{2} \cup \Omega_{3}$ is attractive and invariant for system (1) with the controller (9).

This lemma ensures that the depollution objective is achieved by the controller; the pollution level will always be kept below $S_{\lambda \max }$ once the controller has forced the system into that region. We will now study the behavior of the system in $\Omega_{2}$ and check if Objective 1 is achieved.

Region $\Omega_{2}: D=D_{\max }$ The region $\Omega_{2}$ is defined as

$$
\Omega_{2}=\left\{\left(X_{1}, X_{2}, S_{1}, S_{2}\right) \in\left(\mathbb{R}^{+}\right)^{4} \mid S_{1}+\lambda S_{2} \leq S_{\lambda \min }\right\}
$$

In this region, where $S_{\lambda} \leq S_{\lambda \text { min }}$, system (1) can be rewritten as:

$$
\left\{\begin{array}{l}
\dot{X}_{1}=\left(\mu_{1}\left(S_{1}\right)-\alpha D_{\max }\right) X_{1} \\
\dot{X}_{2}=\left(\mu_{2}\left(S_{2}\right)-\alpha D_{\max }\right) X_{2} \\
\dot{S}_{1}=D_{\max }\left(S_{1 \text { in }}-S_{1}\right)-k_{1} \mu_{1}\left(S_{1}\right) X_{1} \\
\dot{S}_{2}=D_{\max }\left(S_{2 i n}-S_{2}\right)+k_{2} \mu_{1}\left(S_{1}\right) X_{1}-k_{3} \mu_{2}\left(S_{2}\right) X_{2}
\end{array}\right.
$$

In $\Omega_{2}$, we only need to check the evolution of $S_{\lambda}(t)$, which follows the equation (5):

$$
\begin{aligned}
\dot{S}_{\lambda} & =D_{\max }\left(S_{\lambda i n}-S_{\lambda}\right)-\left(k_{1}-\lambda k_{2}\right) \mu_{1}\left(S_{1}\right) X_{1}-\lambda k_{3} \mu_{2}\left(S_{2}\right) X_{2} \\
& \geq D_{\max }\left(S_{\lambda i n}-S_{\lambda \min }\right)-\left(k_{1}-\lambda k_{2}\right) \mu_{1}\left(S_{1}\right) X_{1}-\lambda k_{3} \mu_{2}\left(S_{2}\right) X_{2}
\end{aligned}
$$

From Lemma 1, we know that there exists a finite time $T>0$ after which $X_{1}(t) \leq \frac{S_{1 i n}}{k_{1} \alpha}$ and $X_{2}(t) \leq$ $\frac{T_{2 i n}}{k_{3} \alpha}$. We then have

$\dot{S}_{\lambda} \geq D_{\max }\left(S_{\lambda i n}-S_{\lambda \min }\right)-\left(k_{1}-\lambda k_{2}\right)\left[\max _{S_{1} \leq S_{\lambda \min }} \mu_{1}\left(S_{1}\right)\right] \frac{S_{1 i n}}{k_{1} \alpha}-\lambda k_{3}\left[\max _{S_{2} \leq \frac{S_{\lambda \min }}{\lambda}} \mu_{2}\left(S_{2}\right)\right] \frac{T_{2 i n}}{k_{3} \alpha}$

for all $S_{\lambda} \leq S_{\lambda m i n}$. In order to have $\dot{S}_{\lambda}$ always positive, we impose the following assumption 
Assumption 3 Suppose that

$$
D_{\max }>\frac{\left(k_{1}-\lambda k_{2}\right) \mu_{1}\left(S_{\lambda \min }\right) \frac{S_{1 i n}}{k_{1} \alpha}+\lambda k_{3}\left[\max _{S_{2} \leq \frac{S_{\lambda \min }}{\lambda}} \mu_{2}\left(S_{2}\right)\right] \frac{T_{2 i n}}{k_{3} \alpha}}{S_{\lambda i n}-S_{\lambda \min }}
$$

As $D_{\max }$ is upper-bounded because of equation (4), this assumption can be satisfied by picking the free parameter $S_{\lambda \min }$ small enough. From this expression, we deduce the following lemma:

Theorem 1 Assumptions 1, 2, and 3 ensure that there exists a finite time after which Objective 1 is satisfied by system (1) with controller (9).

This theorem is a consequence of the observations made prior to its statement, which show that all solutions have to leave $\Omega_{2}$ after a finite time, and of Lemma 2 which shows the same thing for $\Omega_{1}$. All solutions then converge to the invariant set $\Omega_{3}$ inside which the depollution objective is achieved. Note that attractivity and invariance of the region of interest is not directly ensured: the solutions first have to converge to the region where (7)-(8) is satisfied (and we have shown that this takes place in finite time), and then we know that $\Omega_{3}$ is attractive and invariant.

\section{SIMULATIONS}

We have implemented controller (9) on model (1). For the simulations, we have used the parameters of the model that were given in (Bernard et al. 2001). We then fixed the following "free" parameters as follows:

$$
S_{\lambda \max }=1.5 ; S_{\lambda \min }=1.3 ; S_{1 \text { in }}=15 ; S_{2 i n}=15 ; \lambda=0.0064 ; D_{\max }=0.5 ; D_{\min }=0.05 .
$$

As can be seen from these parameters, the purpose of the control design is here to steer $S_{\lambda}$ into the interval $[1.3,1.5]$ with a control effort lying in the interval $[0.05,0.5]$. We have considered
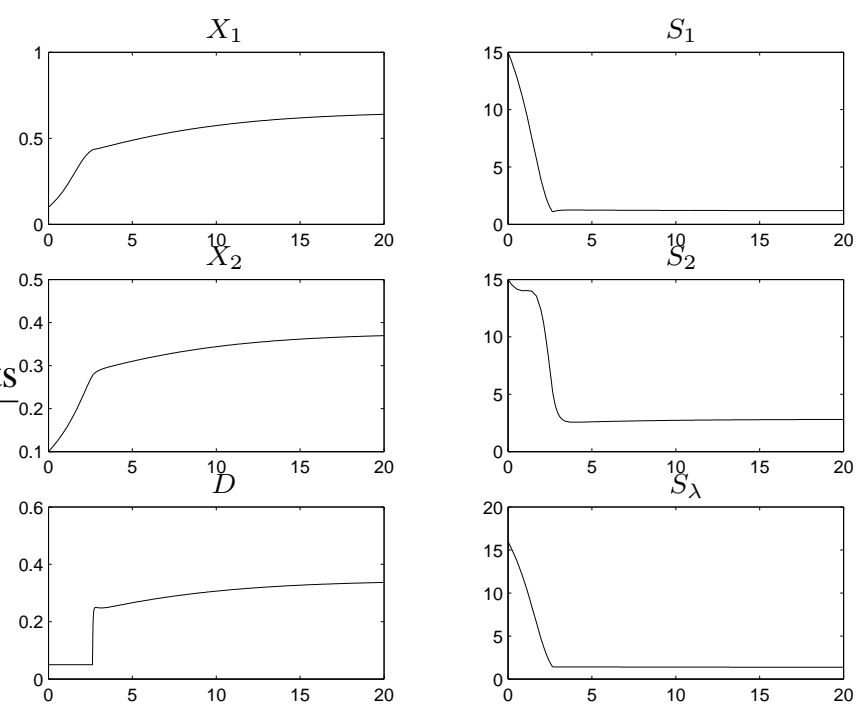

Figure 2: Time evolution of the states, control $D$, and output $S_{\lambda}$ for the control system

$\left(S_{1}, S_{2}, X_{1}, X_{2}\right)(0)=(15,15,0.1,0.1)$ as initial conditions. This set is characterized by a low biomass at the start and a high pollution level in the reactor $\left(S_{\lambda}(0)=15.96\right)$, coming from the large amount of $S_{1}$ in the reactor. The dilution rate is then set at the minimal level during the first two and half days. As can be seen on Figure 2, this forces a decrease of the pollution level $S_{\lambda}, S_{1}$ and $S_{2}$. Simultaneously, the biomasses $X_{1}$ and $X_{2}$ quickly increase. After 3 days, the pollution level settles 
at the desired value, between $S_{\lambda \min }$ and $S_{\lambda \max }$. However, it is interesting to notice that this does not mean that the solution has reached its equilibrium: between day 3 and day 20, we observe a continuing increase of $X_{1}$ and $X_{2}$, coupled with an increase of $D$; indeed, after three days, the reactor is able to treat the wastewater, but the dilution must stay moderate; the subsequent increase of biomass ensures that the plant can handle a higher dilution rate. After that, the equilibrium is reached.

\section{CONCLUSION}

In this paper, we have given a control law for the regulation of a model of anaerobic digestion with two bacteria. We have presented a control that regulates the pollution level: it ensures that the pollution level stays between a minimal and a maximal value while the dilution rate is also fixed between a minimal and a maximal value. No analysis of the actual behavior of the system inside the region where $S_{\lambda}$ belongs to the desired interval has been presented here, but a condition can be given to ensure that the system has a single equilibrium.

Our controller requires that a measure of the pollution level is available online. If it is not the case, we will need to design an observer that will help reconstruct the value of $S_{\lambda}$ from the available observations, namely the methane gaseous flow rate $=k_{6} \mu_{2}\left(S_{2}\right) X_{2}$, and some measures of $S_{\lambda}$ (made with large time intervals in between them). No influent concentration knowledge is required.

Acknowledgment: This work has been supported by the European commission, Information Society Technologies program, Key action I Systems \& Services for the Citizen, contract TELEMAC (IST-2000-28256).

\section{References}

Alcaraz-Gonzalez, V., Harmand, J., Rapaport, A., Steyer, J., Alvarez, V. G. and Ortiz, C. P. (2002). Software sensors for highly uncertain WWTPs : a new approach based on interval observers, Water Research 36: $2515-2524$.

Antonelli, R., Harmand, J., Steyer, J. and Astolfi , A. (2003). Set-point regulation of an anaerobic digestion process with bounded output feedback, IEEE Tr. on Cont. Syst. 11 (4): 495-504.

Bastin, G. and Dochain, D. (1990). On-line Estimation and Adaptive Control of Bioreactors, Elsevier.

Batstone, D. J., Keller, J., Angelidaki, I., Kalyuzhnyi, S., Pavlostathis, S., Rozzi, A., Sanders, W., Siegrist, H. and Vavilin, V. (2002). The IWA anerobic digestion model no.1 (ADM1), Wat. Sci. Tech. 45 (10): 65-73.

Bernard, O., Hadj-Sadok, Z., Dochain, D., Genovesi, A. and Steyer, J. P. (2001). Dynamical model development and parameter identifi cation for an anaerobic wastewater treatment process, Biotech. Bioeng. 75: 424-438.

Genovesi, A., Harmand, J. and Steyer, J. (1999). A fuzzy logic based diagnosis system for the on-line supervision of an anaerobic digestor pilot-plant, Biochemical Engineering Journal 3: 171-183.

Mailleret, L., Bernard, O. and Steyer, J. (2003). Robust regulation of anaerobic digestion processes, Wat. Sci. Tech. 48-6: 87-94.

Mailleret, L., Bernard, O. and Steyer, J. (2004). Nonlinear adaptive control for bioreactors with unknown kinetics, Automatica 40(8): 1379-1385.

Perrier, M. and Dochain, D. (1993). Evaluation of control strategies for anaerobic digestion processes, International Journal on Adaptive Control and Signal Processing 7: 309-321.

Punal, A., Rodriguez, J., Franco, A., Carrasco, E., Roca, E. and Lema, J. (2000). Advanced monitoring and control of anaerobic wastewater treatment plants: Diagnosis and supervision by a fuzzy-based expert system, Wat. Sci. Tech. 43 (7): 191-198. 